\title{
High-Resolution Imaging of the Optic Nerve and Retina in Optic Nerve Hypoplasia
}

Anastasia Pilat, PhD,* Daniel Sibley, BMBS,* Rebecca J. McLean, MSc, Frank A. Proudlock, PhD, Irene Gottlob, MD

Purpose: To investigate the optic nerve and macular morphology in patients with optic nerve hypoplasia $(\mathrm{ONH})$ using spectral-domain optical coherence tomography (SD OCT).

Design: Prospective, cross-sectional, observational study.

Subjects: A total of 16 participants with $\mathrm{ONH}$ (10 female and 6 male; mean age, 17.2 years; 6 bilateral involvement) and 32 gender-, age-, ethnicity-, and refraction-matched healthy controls.

Methods: High-resolution SD OCT (Copernicus [Optopol Technology S.A., Zawiercie, Poland], $3 \mu \mathrm{m}$ resolution) and handheld SD OCT (Bioptigen Inc [Research Triangle Park, NC], $2.6 \mu \mathrm{m}$ resolution) devices were used to acquire horizontal scans through the center of the optic disc and macula.

Main Outcome Measures: Horizontal optic disc/cup and rim diameters, cup depth, peripapillary retinal nerve fiber layer (RNFL), and thickness of individual retinal layers in participants with $\mathrm{ONH}$ and in controls.

Results: Patients with $\mathrm{ONH}$ had significantly smaller discs $(P<0.03$ and $P<0.001$ compared with unaffected eye and healthy controls, respectively), horizontal cup diameter $(P<0.02$ for both), and cup depth $(P<0.02$ and $P<0.01$, respectively). In the macula, significantly thinner RNFL (nasally), ganglion cell layer (GCL) (nasally and temporally), inner plexiform layer (IPL) (nasally), outer nuclear layer (ONL) (nasally), and inner segment (centrally and temporally) were found in patients with ONH compared with the control group $(P<0.05$ for all comparisons). Continuation of significantly thicker GCL, IPL, and outer plexiform layer in the central retinal area (i.e., foveal hypoplasia) was found in more than $80 \%$ of patients with $\mathrm{ONH}$. Clinically unaffected fellow eyes of patients with $\mathrm{ONH}$ showed mild features of underdevelopment. Visual acuity and presence of septo-optic dysplasia were associated with changes in GCL and IPL. Sensitivity and specificity for the detection of ONH based on disc and retinal optical coherence tomography (OCT) parameters were $>80 \%$.

Conclusions: Our study provides evidence of retinal changes in $\mathrm{ONH}$. In addition to thinning of retina layers mainly involving the RNFL and GCL, signs reminiscent of foveal hypoplasia were observed in patients with ONH. Optic nerve and foveal parameters measured using OCT showed high sensitivity and specificity for detecting $\mathrm{ONH}$, demonstrating their useful for clinical diagnosis. Ophthalmology 2015;m:1-10 (c) 2015 by the American Academy of Ophthalmology. This is an open access article under the CC BY-NC-ND license (http://creativecommons. org/licenses/by-nc-nd/4.0/).

Supplemental material is available at www.aaojournal.org.

Optic nerve hypoplasia (ONH) is a nonprogressive congenital abnormality of the optic nerve characterized by up to a $90 \%$ reduction in the number of axons in histologic samples. ${ }^{1}$ Optic nerve hypoplasia has a prevalence estimated between 7.1 and 10.9/100 000 children per year. ${ }^{2,3}$ It can present as an isolated entity in 1 or both eyes, or can coexist with other central nervous system abnormalities. The most well-documented association is septo-optic-dysplasia (SOD). Septo-optic-dysplasia, also known as de Morsier syndrome, consists of the triad of $\mathrm{ONH}$, midline brain abnormalities, including absent septum pellucidum, hypothalamic dysfunction, and endocrine abnormalities.

The cause of ONH is not fully understood, and multiple causative etiologies may produce clinically indistinguishable phenotypes. The majority of cases are sporadic. However, there have been familial cases of SOD with $\mathrm{ONH}$ described with mutations in $\mathrm{HESX}^{4}$ and SOX2. ${ }^{5}$ Optic nerve hypoplasia has been associated with abnormal intrauterine development, for example, in maternal diabetes $^{6}$ or fetal alcohol syndrome.

Optic nerve hypoplasia is a clinical diagnosis; visualization of the disc reveals an abnormally small optic nerve head, characteristically pale or grey in color. A "double-ring sign" is often described, consisting of a normal junction between the sclera and the lamina cribrosa (outer ring) and an abnormal extension of the retina and pigment epithelium over the outer portion of the lamina cribrosa (inner ring). Vascular abnormalities are described, frequently vascular tortuosity, ${ }^{8}$ although abnormally straight vessels with reduced branching have been documented.

Spectral-domain optical coherence tomography (OCT) is a noninvasive, noncontact imaging modality that produces in vivo images comparable to histologic samples ${ }^{10}$ with good reproducibility. ${ }^{11}$ Little is known about the morphologic changes of the optic disc in ONH. A histopathology study performed by Mosier et $\mathrm{al}^{12}$ on 1 patient with $\mathrm{ONH}$ 


\section{Ophthalmology Volume - Number $\mathbf{n}$, Month 2015}

showed thinning of the retinal nerve fiber layer (RNFL) and ganglion cell reduction. These findings were confirmed by other researchers. ${ }^{13,14}$ To date, there are only a few articles investigating the in vivo eye morphology in $\mathrm{ONH}$ using optical coherence tomography (OCT) ${ }^{15,16}$ In 2013, Moon and Park ${ }^{16}$ described RNFL thinning and thinning of the structures located between the posterior boundary of the RNFL and the posterior boundary of the outer plexiform layer (OPL) of the retina measured together in 1 patient with ONH compared with the healthy eye using SD OCT. There are no investigations about possible macular changes associated with $\mathrm{ONH}$. A previous study using fundus photography has found no relationship between the size of the optic nerve and the visual acuity in patients with ONH. ${ }^{13}$

The aim of this study is to perform the first crosssectional observational study to characterize the optic nerve and macular morphology in a series of patients with various degrees of $\mathrm{ONH}$ using high-resolution SD OCT. We also explore the clinical utility of SD OCT in detecting $\mathrm{ONH}$ and correlate associated changes with the visual outcome and presence of SOD.

\section{Methods}

\section{Subjects}

Sixteen patients with $\mathrm{ONH}$ (10 female and 6 male; mean age, 17.2 years; standard deviation \pm 16.22 ) and 32 gender-, age-, ethnicity-, and refraction-matched healthy controls were included in this prospective observational study. Six patients demonstrated clinically bilateral involvement. Eyes were divided into 2 subgroups: eyes with (affected, $\mathrm{n}=22$ ) and without (unaffected, $\mathrm{n}=10$ ) clinically detected $\mathrm{ONH}$.

All participants underwent a standard ophthalmologic examination, including best-corrected visual acuity, refraction, orthoptic examination, slit-lamp examination, and dilated fundoscopy (Table 1, available at www.aaojournal.org). When nystagmus was present, eye movements were recorded (EyeLink 1000, SR Research Ltd, Osgoode, Canada) under binocular conditions and either eye occluded. Nystagmus waveforms were observed for infantile nystagmus (i.e., accelerating or sinusoidal slow phases) and manifest latent nystagmus (decelerating waveforms with the slow phase directed toward the occluded eye). Two participants (aged 12 and 16 years) were too young to cooperate with eye movement recordings, and nystagmus type was determined clinically (manifest latent nystagmus if change in direction on covering).

The diagnosis of ONH was established on the basis of clinical examination. Patients with $\mathrm{ONH}$ had no other known ophthalmic pathology. Magnetic resonance imaging of the brain and orbits was performed in 10 of 16 patients ( 6 adult patients preferred not to undergo magnetic resonance imaging as part of the examination) to determine the presence of SOD. Five of 16 patients did not have nystagmus. All 5 patients had unilateral $\mathrm{ONH}$.

A total of 32 gender-, age-, ethnicity-, and refraction-matched healthy controls with best-corrected visual acuity of 0.2 logarithm of the minimum angle of resolution (logMAR) or better, normal visual fields, and intraocular pressure (when possible to assess, because some participants were too young to cooperate) were included. The control group had no known eye pathology, systemic disease, or previous intraocular surgery. The study adhered to the tenets of the Declaration of Helsinki and was approved by the local ethics committee. Informed consent was obtained from all participants or their parents or guardians.

\section{Optical Coherence Tomography}

Ultra-high-resolution spectral-domain OCT (Copernicus; Optopol Technology S.A., Zawiercie, Poland) was used to acquire tomograms with a wavelength of $850 \mathrm{~nm}$ and a theoretic axial resolution of $3.0 \mu \mathrm{m}(7 \times 7 \times 2 \mathrm{~mm}, 75 \mathrm{~B}$-scans, $743 \mathrm{~A}$-scans per B-scan, fixation target set to image the optic disc) in 9 older participants in whom we could achieve a stable head position to obtain goodquality scans. In 7 participants (aged $1-7$ years), SD OCT images were obtained using a handheld device (HH-OCT, Envisu, Bioptigen Inc, Research Triangle Park, NC) with a wavelength of $840 \mathrm{~nm}$ and theoretic axial resolution of $2.4 \mu \mathrm{m}(10 \times 10 \times 2.46 \mathrm{~mm}$, $100 \mathrm{~B}$-scans and $500 \mathrm{~A}$-scans per B-scan). Individual horizontal B-scans were analyzed as opposed to automated volumetric analysis because of the presence of nystagmus in 10 patients. Because of the rapid acquisition time for individual B-scans $(14.3 \mathrm{~ms}$ for Copernicus and $15.6 \mathrm{~ms}$ for Bioptigen Inc), distortion of individuals' B-scans due to nystagmus was minimal.

The consistency of measurements between the 2 devices was checked by comparing measurements from 15 adults with both devices. Interclass correlation coefficients for key parameters were all $>0.8$ (rim diameter $=0.91$; cup diameter $=0.93$; retinal thickness $=0.94,0.93$, and 0.95 for nasal, central, and temporal, respectively; GCL, central $=0.83$ ).

\section{Optic Nerve Head Analysis}

A flattened B-scan through the deepest point of the optic nerve cup was used for $\mathrm{ONH}$ analysis. Quantitative OCT analysis was conducted in a semiautomated manner using an ImageJ macro (National Institutes of Health, Bethesda, MD, available at: http:// rsbweb.nih.gov/ij/) by the same investigator (AP) for all scans.

On the horizontal tomograms, the edges of the retinal pigment epithelium (RPE) (optic disc margins), the position of the internal limiting membrane, and the RNFL position were marked manually (Fig 1A). The cup diameter (using a cup offset $150 \mu \mathrm{m}$ anteriorly to the disc axis), cup maximal depth, and horizontal rim size (distance between horizontal disc and cup diameters) and temporal and nasal height (between the horizontal cup diameter level and the RNFL, limited on the periphery by the disc margins) were measured automatically by the macro. Peripapillary RNFL thickness was measured in a region from 1200 to $1600 \mu \mathrm{m}$ on both sides of the center of the cup (same as the default setting in the Optopol automated analysis).

\section{Foveal Analysis}

A central horizontal flattened B-scan was selected at the deepest point of the foveal pit where the outer segment (OS) of photoreceptors was thickest, indicating specialization (i.e., elongation) of photoreceptors at the pit. If there was no clear foveal pit, the center of the macula was identified as the point with greatest extent of thinning of the inner retinal layers and doming of the outer nuclear layer (ONL).

Detailed SD OCT analysis was conducted in a semiautomated manner using an ImageJ macro with the retinal layer borders positioned manually by locating points that were fitted with a spline fit. The borders were used to calculate thickness measurements of the RNFL, ganglion cell layer (GCL), inner plexiform layer (IPL), inner nuclear layer (INL), OPL, ONL, inner segment (IS), OS, contact cylinder, and RPE layers (Fig 1B). The position of the retinal layers was measured across the whole scan. For statistics, the thickness measurements in the central point, paracentral area (averaged thickness of each layers from $250 \mu \mathrm{m}$ nasally to $250 \mu \mathrm{m}$ temporarily from the center), and nasal/ temporal areas (averaged thickness of each layers from 500 to 


\section{Pilat et al • Optical Coherence Tomography in $\mathrm{ONH}$}
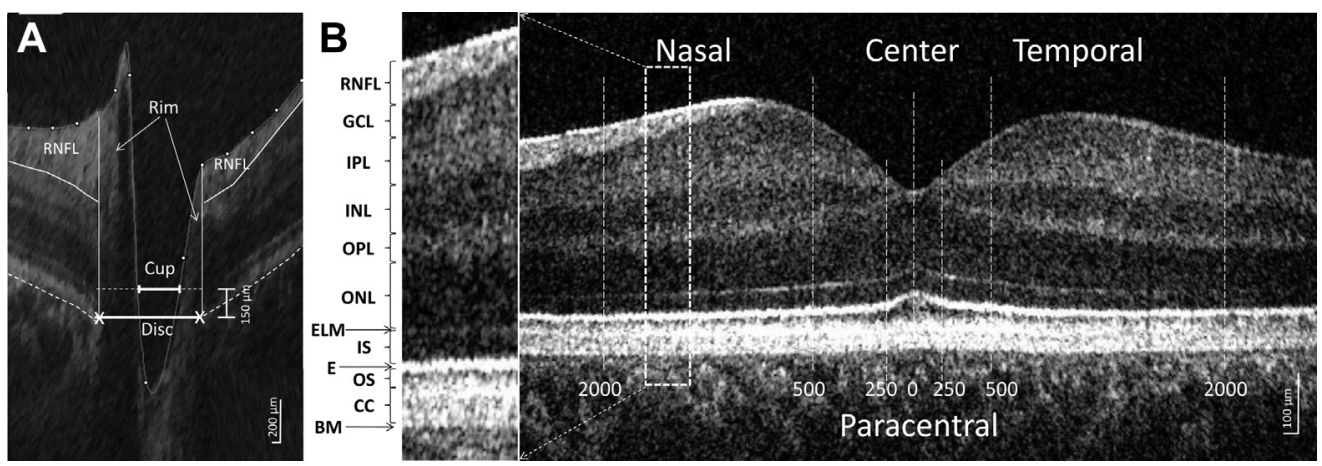

Figure 1. Horizontal spectral-domain optical coherence tomography (SD OCT) B-scan images of the (A) optic nerve head and (B) macula area of a control subject. A, An OCT scan of the optic nerve head where the disc diameter was defined as the distance between the edges of retinal pigment epithelium (RPE); cup diameter was measured as the length of the line parallel to the disc diameter in $150 \mu \mathrm{m}$ anteriorly to the disc and limited by the internal limiting membrane; nasal and temporal rims were calculated as the difference between the disc and cup edges; and maximal cup depth was measured as a perpendicular line between the cup diameter and the deepest point of the cup, retinal nerve fiber layer (RNFL), was delineated manually. B, The position of the different retinal layers $(l e f t)$ in an OCT scan of the macular area (right). The thickness of the layers was measured in the center of the fovea, in the paracentral area (from $250 \mu \mathrm{m}$ nasally to $250 \mu \mathrm{m}$ temporally), and nasally and temporally (from 500-2000 $\mu \mathrm{m}$ ). BM $=$ Bruch's membrane; CC $=$ contact cylinder; $\mathrm{E}=$ ellipsoid; $\mathrm{ELM}=$ external limiting membrane; $\mathrm{GCL}=$ ganglion cell layer; $\mathrm{INL}=$ inner nuclear layer; $\mathrm{IPL}=$ inner plexiform layer; IS $=$ inner segment; $\mathrm{ONL}=$ outer nuclear layer; $\mathrm{OPL}=$ outer plexiform layer; $\mathrm{OS}=$ outer segment.

$2000 \mu \mathrm{m}$ from the center, nasally and temporarily, respectively) were used.

\section{Statistical Analysis}

Statistical analysis was performed using SPSS software version 16.0 (SPSS, Inc, Chicago, IL). Optic nerve head and macular parameters were normally distributed as determined by the ShapiroWilk test. The parameters of the $\mathrm{ONH}$ and macula were analyzed using a univariate mixed linear model that included group as a fixed factor and corrected for refractive error. Bonferroni correction was used for post hoc multiple comparisons (comparing affected eyes and unaffected eyes in participants with $\mathrm{ONH}$ and controls). Correlations between optic nerve and macular parameters were made using Pearson's correlation. Optic nerve and macular parameters were correlated with visual acuity. We also analyzed whether the presence of SOD was associated with optic nerve parameter changes using Spearman's rank correlation. $P \leq 0.05$ was considered statistically significant.

The sensitivity and specificity of optic nerve and macula parameters for $\mathrm{ONH}$ detection were calculated by defining positive and negative results using fixed thresholds for each optic disc and macular parameter that demonstrated statistically significant changes in $\mathrm{ONH}$.

\section{Results}

\section{Optic Nerve Head Morphology}

In Figure 1A, the fundus images and OCT B-scans of a patient are shown with right $\mathrm{ONH}$ and a left eye with a clinically unaffected optic nerve. The OCT scans showed the coexistence of optic nerve head drusen (ONHD) as an incidental finding in 3 of the 16 patients $(18.75 \%)$. Drusen appeared as hyper-reflective round bodies above the edges of the RPE (Fig 2B). The ONHD were seen in both eyes (with and without ONH). Patients with ONHD were excluded from analysis because ONHD can be associated with retinal changes. ${ }^{17}$

On visual inspection of the optic nerve head scans of eyes affected with ONH (examples in Figs $2 \mathrm{~A}$ and 3), the optic discs and cups were clearly smaller compared with those in control subjects.

Mean optic nerve head parameters in clinically affected and unaffected eyes of patients with $\mathrm{ONH}$ and healthy controls are illustrated schematically in Figure 4. Individual values are shown in Figure 5. Cup diameters and depths, and disc diameters were profoundly smaller in affected eyes in patients with $\mathrm{ONH}$ compared with both clinically unaffected eyes in patients with ONH (percentage differences: 103\%, 96\%, 46\%, respectively; $P=0.02, P=0.02, P=0.03$, respectively) and eyes in healthy controls (percentage differences: 115\%, 103\%, $65 \%$, respectively; $P=0.003, P=0.01, P=0.002$, respectively).

Although rim sizes in patients with $\mathrm{ONH}$ were apparently small on visual inspection, analysis of the rim cross-sectional areas and asymmetry in the nasal and temporal cross-sectional areas showed no statistical differences among the 3 groups. As can be seen in Figure 4, clinically unaffected eyes from patients with $\mathrm{ONH}$ had smaller disc/cup diameters and cup depth compared with eyes of healthy controls; however, this difference was not significant (Fig 5).

Sensitivity and specificity for the detection of ONH based on SD OCT disc parameters were $85.7 \%$ and $92.8 \%$ for disc diameter, $85.7 \%$ and $90.9 \%$ for cup diameter, and $85.7 \%$ and $91.6 \%$ for cup depth, respectively (using thresholds of 1200, 550, and $200 \mu \mathrm{m}$, respectively).

Peripapillary RNFL was significantly thinner in the affected eye of patients with ONH compared with controls, with similar differences being observed in both nasal and temporal RNFL (54\% and $68 \%$ of control values, respectively) (Fig 6). The RNFL thicknesses for the clinically unaffected eyes of patients with $\mathrm{ONH}$ were approximately midway between thicknesses in affected eyes of patients with $\mathrm{ONH}$ and controls. We found no significant difference in peripapillary RNFL between the 2 eyes in patients with bilaterally hypoplastic discs for any optic disc parameter.

Correlation analyses were performed for all optic disc and RNFL parameters in the affected eye correlating the following:

Optic disc and RNFL parameters with each other: A significant positive correlation was detected between peripapillary RNFL thickness in the nasal area and both the horizontal diameter of the $\operatorname{rim}(r=0.80, P=0.031)$ and the size of the nasal rim area $(r=$ $0.80, P=0.030)$. 
Ophthalmology Volume a, Number a, Month 2015

A

Right eye

Left eye

Patient with Right Eye Optic Nerve Hypoplasia
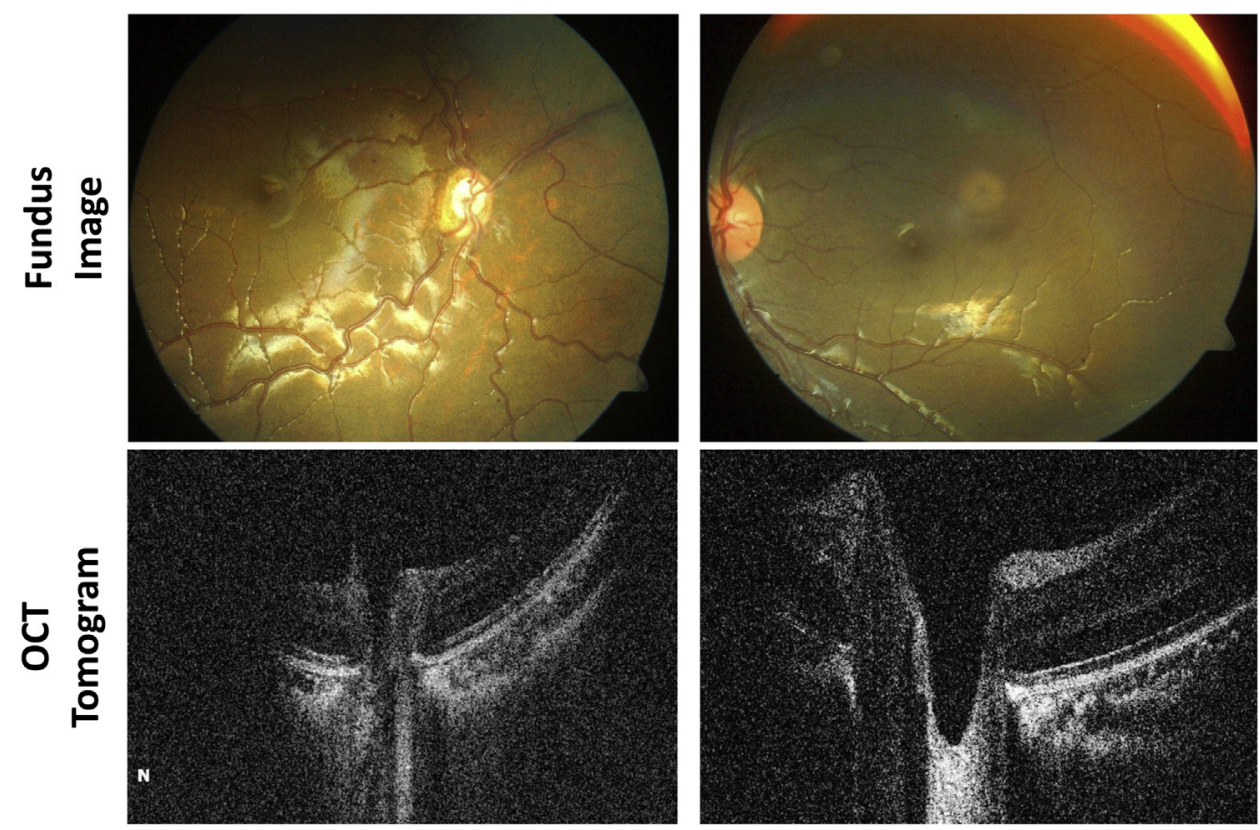

B

Patient with Bilateral Optic Nerve Head Drusen and Left Eye Optic Nerve Hypoplasia

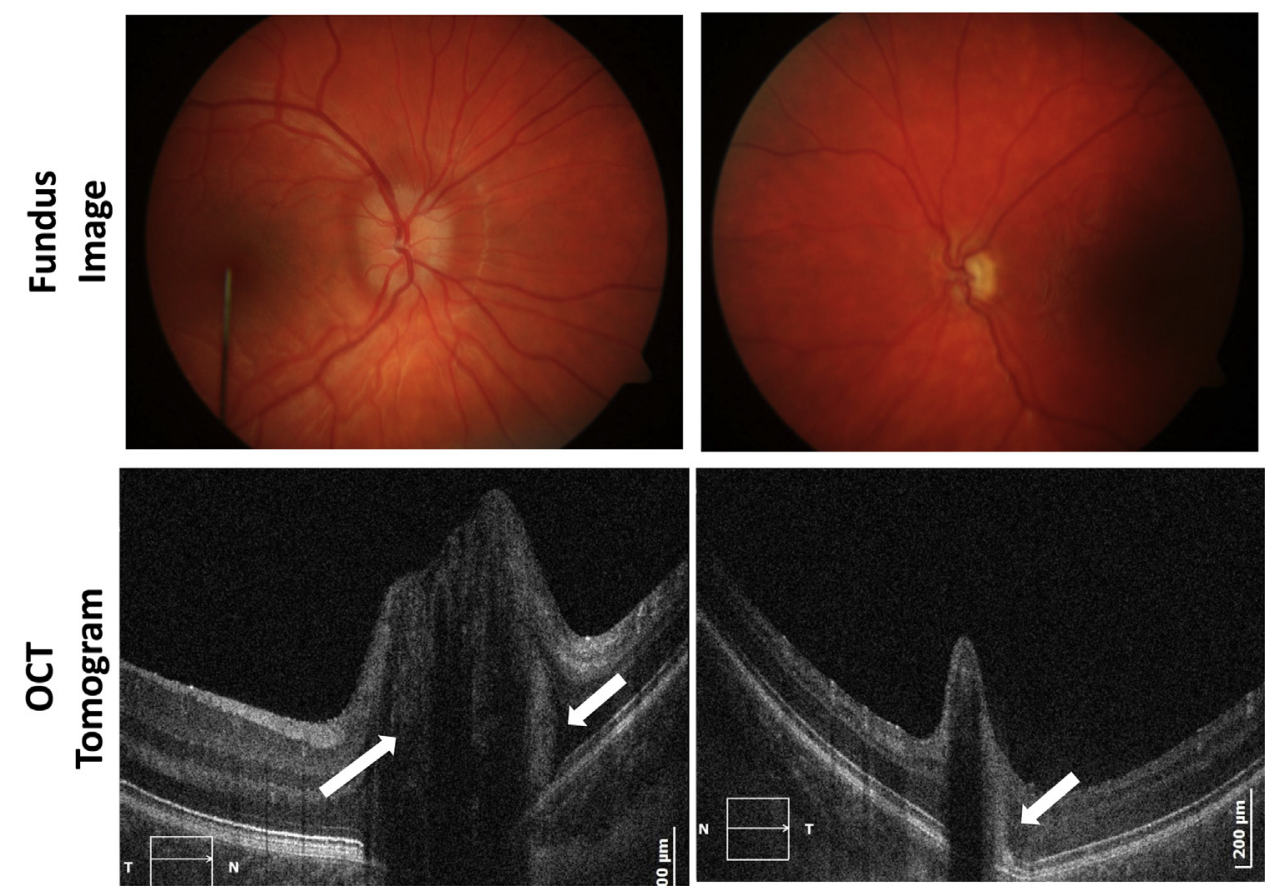

Figure 2. Fundus images (top) and spectral-domain optical coherence tomography (SD OCT) horizontal B-scans (bottom) through the center of the disc in (A) a patient with right eye optic nerve hypoplasia $(\mathrm{ONH})$ (patient 1, Table 1, available at www.aaojournal.org) and (B) bilateral optic nerve head drusen (ONHD) with ONH in the left eye (patient 8, Table 1, available at www.aaojournal.org). Arrows indicate the position of the drusen above the edges of the RPE. The disc size of the left eye with both ONHD and ONH is considerably smaller than in the unaffected eye. 


\section{Pilat et al · Optical Coherence Tomography in $\mathrm{ONH}$}

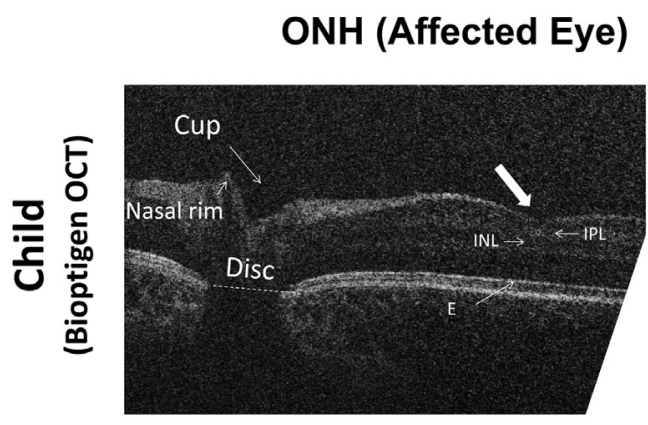

Healthy Controls

\section{Optic Nerve Head}

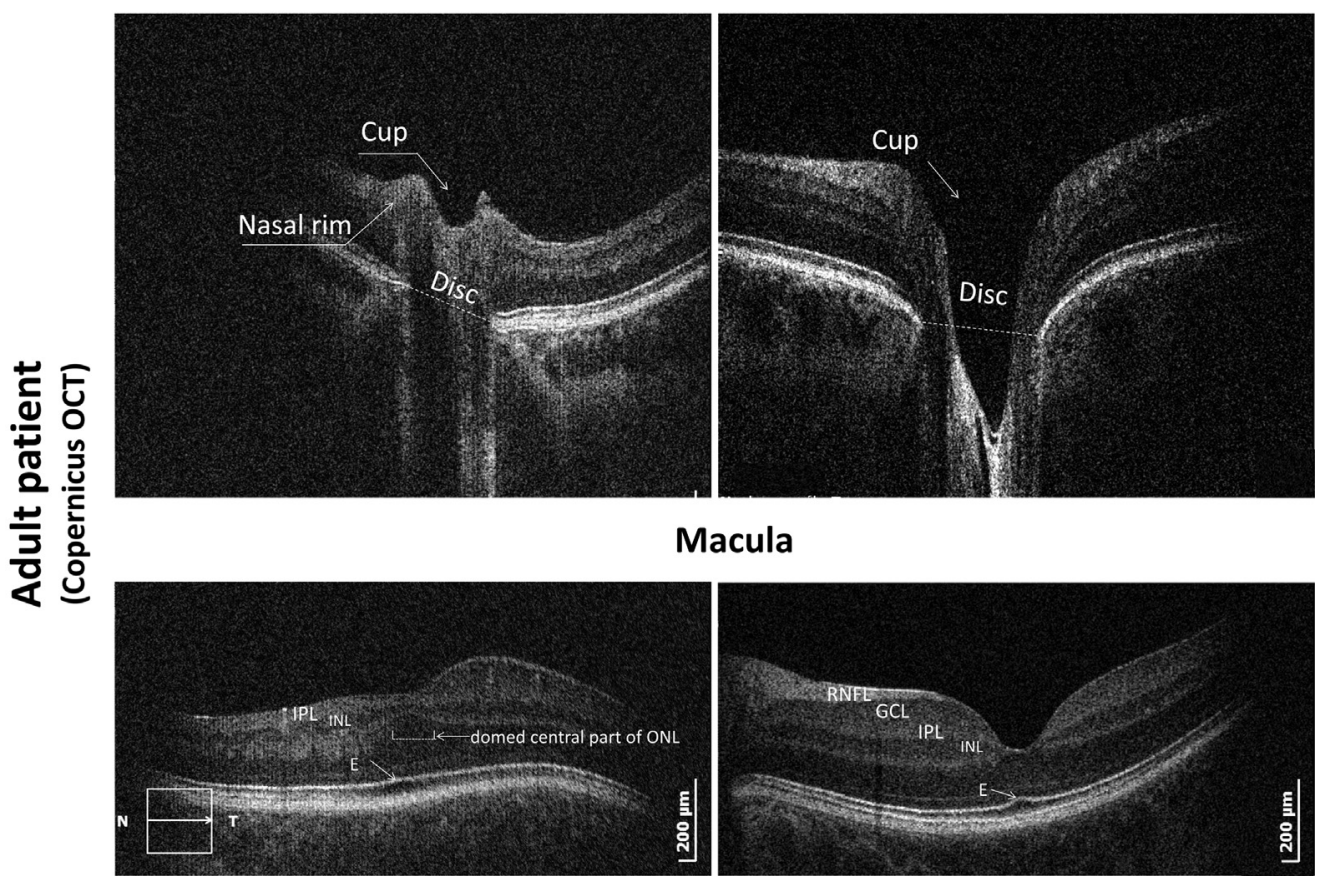

Figure 3. Examples of spectral-domain optical coherence tomography (SD OCT) of affected left eyes of the patients with optic nerve hypoplasia (ONH) (left) and age-, gender-, ethnicity-, and refraction-matched healthy controls (right). The optic nerve head scans of patients with ONH demonstrated small disc and cup diameters, and cup depth. The horizontal macula scans of the patients with ONH (bottom left and top left) demonstrate a thin retina, flat foveal pits, a thin retinal nerve fiber layer (RNFL) and ganglion cell layer (GCL), continuation of the inner plexiform layer (IPL) and inner nuclear layer (INL), a domed central area of ONL, and absence of the upward deflection of the ellipsoid line in the center of the fovea in patients with ONH. E = ellipsoid.

Clinically unaffected eye: A significant positive correlation was detected for cup depth $(r=0.78, P=0.04)$.

Visual acuity, SOD, nystagmus: No significant correlations were observed between visual acuity or SOD and optic disc and RNFL parameters. Neither the presence of nystagmus nor the type of waveform (infantile nystagmus or manifest latent nystagmus) was correlated to optic disc/RNFL parameters or mono/bilateral involvement.

\section{Foveal Morphology}

Visual inspection of the horizontal macular SD OCT scans of affected eyes revealed a thinner parafoveal retina overall, mainly because of a thin RNFL or thin or absent GCL, which led to a flatter foveal pit. In the fovea, continuation of the GCL, IPL, and INL was observed. There was an absence of the upward deflection of the ellipsoid line in the center of the fovea normally present because of elongation of cones caused by increased packing. The ONL in the foveal area had a more domed appearance in affected ONH eyes compared with healthy controls (as can be seen by comparing bottom left and right images in Figure 3, and comparing the top and bottom schematic representations in Fig 7A). The pattern of continuation of inner layers and domed ONL was observed in 18 of 22 eyes with $\mathrm{ONH}$, with neither feature observed in the remaining 4 patients. Consequently, this pattern gave a sensitivity of $81.8 \%$ and specificity of $100 \%$ for $\mathrm{ONH}$ diagnosis.

Quantitative analysis showed that affected eyes had significantly thicker central and paracentral $(500 \mu \mathrm{m}$ diameter $)$ areas compared with both clinically unaffected eyes $(11 \%$ and $16 \%$, respectively, $P<0.05)$ and controls $(15 \%$ and $18 \%$, respectively, $P<0.05)$. In contrast, the nasal retina was thinner in the affected eyes compared with the clinically unaffected eyes $(26 \%, P<0.05)$ and controls $(33.2 \%, P<0.05)$. The temporal area was also significantly thinner in the affected eye of patients with $\mathrm{ONH}$ compared with healthy participants $(11 \%, P=0.005)$.

Increased central retinal thickness in the affected eyes of patients with $\mathrm{ONH}$ compared with clinically unaffected eyes and controls was mainly due to changes in the GCL, IPL, and OPL 


\section{ONH (Affected Eye)}

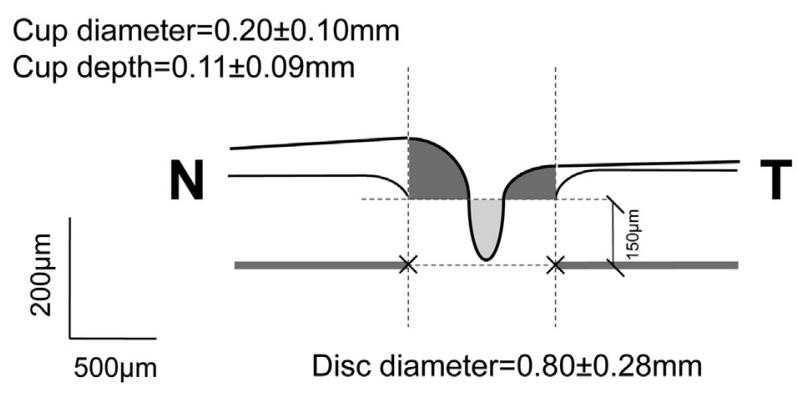

\section{ONH (Unaffected Eye)}

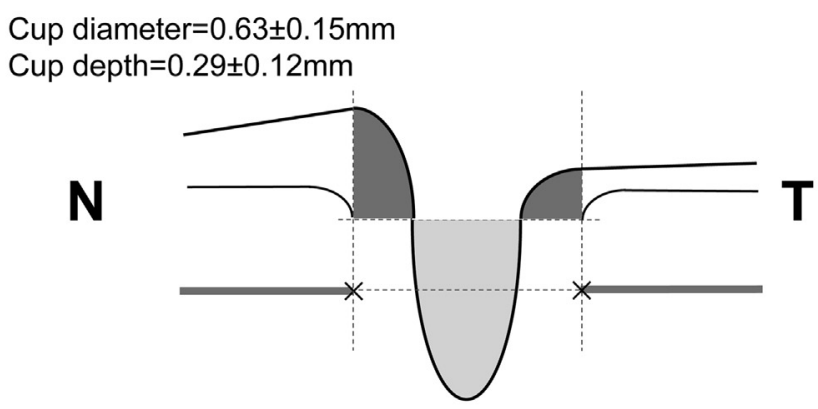

Disc diameter $=1.30 \pm 0.39 \mathrm{~mm}$

\section{Healthy Control}

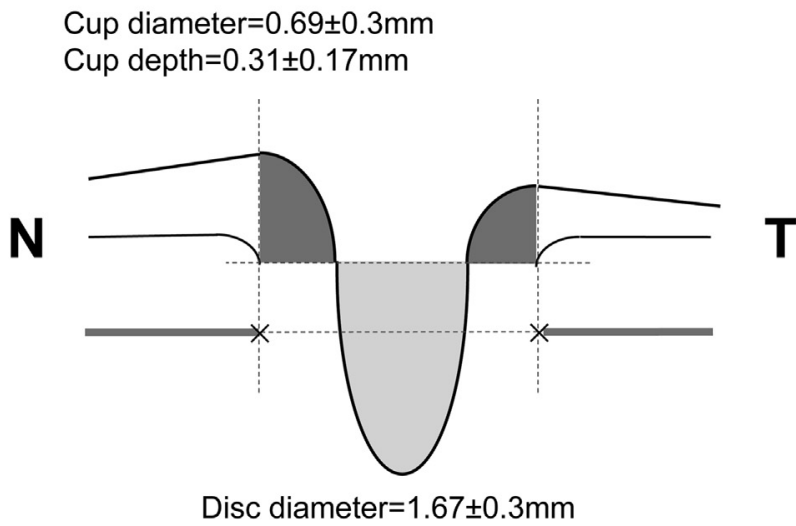

Figure 4. Cross-sectional schematic diagrams representing mean values of optic nerve head parameters of patients with optic nerve hypoplasia $(\mathrm{ONH})$ (affected and clinically unaffected eyes) and controls. Upper horizontal dotted lines represent horizontal offset $(150 \mu \mathrm{m})$ used to determine cup diameters, and lower horizontal dotted lines indicate disc horizontal diameters. Vertical dotted lines show margins of rim areas. Mean $( \pm$ standard error) values of parameters that were statistically significant are given. $\mathrm{N}=$ nasal; $\mathrm{T}=$ temporal
(Fig 7B). Thinning of the nasal retina in affected eyes of patients with ONH was primarily due to thinning of the GCL, with RNL, IPL, and ONL also being affected. The GCL was also thinner in the temporal retina in affected eyes of patients with $\mathrm{ONH}$. Of note, the IS was significantly thinner in affected eyes of patients with $\mathrm{ONH}$ in both central and temporal areas.

Coefficients of data variability of the thicknesses of the layers were between 10.0 and 36.1 (Table 2, available at www.aaojournal.org). A higher coefficient was measured for the RNFL and GCL (36.1 and 24.3 , respectively, in the $\mathrm{ONH}$ group in central area) because they were the thinnest layers in the macula. The sensitivity and specificity for the detection of $\mathrm{ONH}$ were $88.8 \%$ and $100 \%$ for nasal GCL and $77.7 \%$ and $84.6 \%$ for temporal GCL, respectively (using thresholds of $35 \mu \mathrm{m}$ and $30 \mu \mathrm{m}$ for average measurements, respectively).

Correlation analyses were performed for all foveal parameters in the affected eye correlating the following:

Optic disc and RNFL parameters: The horizontal disc diameter showed a significant positive correlation with nasal IPL and temporal IS thickness $(P<0.01$ for both), and a significant negative correlation with central IPL thickness $(P=0.003)$. Consequently, smaller disc diameters were associated with greater retinal thinning in the nasal and temporal areas and continuation of inner retinal layers in the central retina. The horizontal rim diameter in affected eyes showed a significant correlation with nasal RNFL thickness, temporal ONL thickness, and temporal and nasal GCL thickness $(P<0.05$ for all). The horizontal cup diameter was negatively correlated with central IPL and OPL thicknesses $(P=0.001$ and $P=0.02$, respectively). Cup depth showed significant negative correlations with the thickness of the retinal layers in the central area only (GCL: $P=0.03$; IPL: $P<0.001$; OPL: $P=0.01$ ).

Clinically unaffected eye: Only positive correlations were observed for macular parameters in nasal RNFL $(r=0.98, P=$ $0.03)$ and paracentral IPL $(r=1.00, P=0.001)$.

Visual acuity: There was a positive correlation between visual acuity (using $\log$ MAR visual acuity, where higher values indicate poorer vision) and temporal and nasal GCL thickness $(P=0.03$ and $P=0.002$, respectively) and nasal $\operatorname{IPL}(P=0.02)$.

SOD: The presence of SOD was negatively correlated with nasal GCL thickness $(P=0.02)$ and positively correlated with central OPL $(P=0.009)$.

Nystagmus: There were no significant correlations.

\section{Discussion}

The majority of histopathologic studies investigating the eye structure in patients with ONH report thinning of the RNFL and reduction of the GCL. ${ }^{12,13,18}$ To date, the potential of SD OCT to assess optic nerve and macula morphology in patients with ONH in vivo has been largely unexplored. In 2013, Moon and Park ${ }^{16}$ confirmed previous histopathology data in 1 patient with $\mathrm{ONH}$ compared with the healthy eye using SD OCT. In this study we have recruited a substantial number of patients with ONH and report new OCT findings of ONH and retinal changes. We demonstrate that OCT can be used in clinical practice in cases with diagnostic uncertainty to clarify the presence of $\mathrm{ONH}$.

\section{Optic Nerve Head Changes}

Our results indicate that patients with $\mathrm{ONH}$ showed significantly smaller horizontal disc diameters, horizontal cup diameters, and cup depths compared with the unaffected 


\section{Pilat et al • Optical Coherence Tomography in $\mathrm{ONH}$}

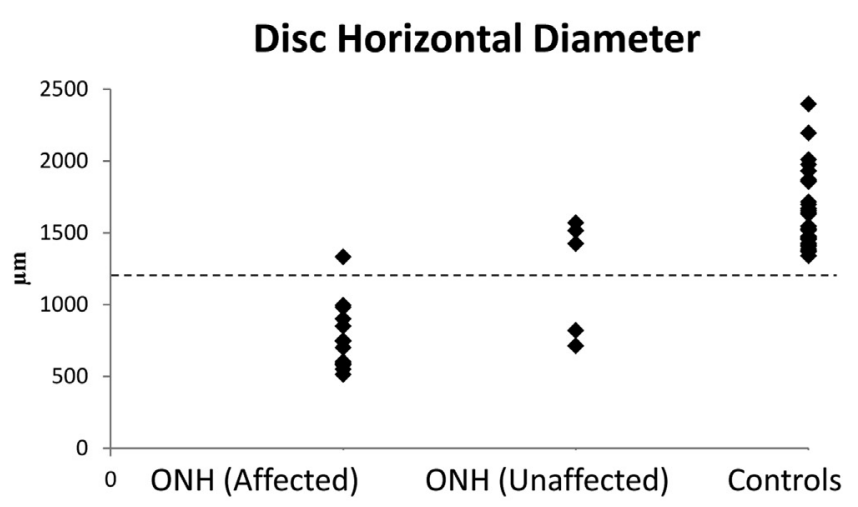

Rim Horizontal Diameter
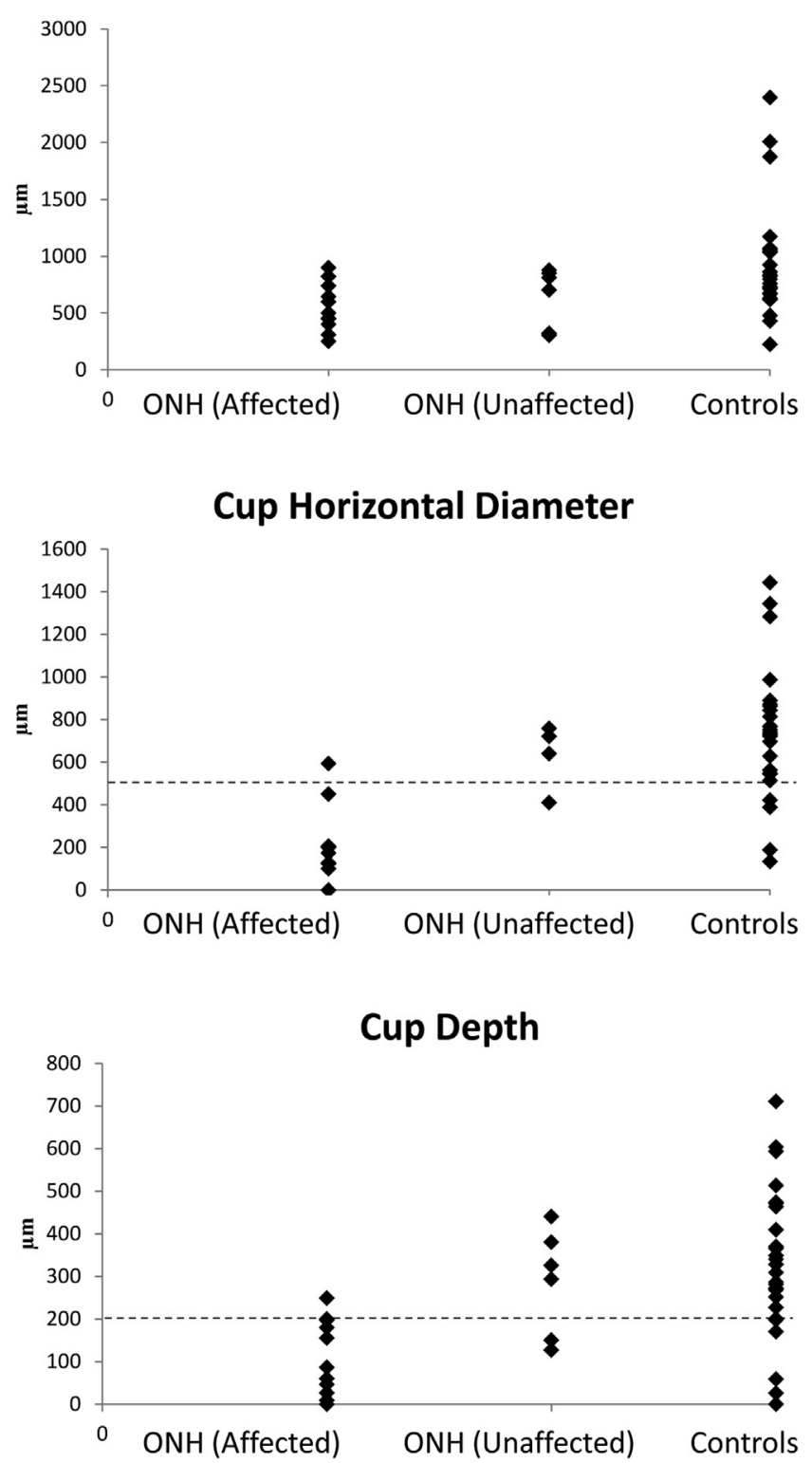

Figure 5. Distribution of optic nerve head parameters $(\mu \mathrm{m})$ in patients with optic nerve hypoplasia $(\mathrm{ONH})$ in affected/clinically unaffected eyes and healthy controls. Horizontal dotted line shows the threshold for optic disc hypoplasia detection in parameters that were highly sensitive and specific. eyes and eyes of healthy controls $(P<0.05)$. All 3 parameters showed sensitivity and specificity of $>85 \%$ and $>90 \%$, respectively, indicating that OCT parameters of the optic nerve head can be used in clinical practice to confirm suspected diagnosis of $\mathrm{ONH}$.

There are several theories for the cause of maldevelopment of the optic nerve in ONH. One hypothesis suggests that failure of GCL differentiation leads to RNFL reduction, one of the features of ONH. ${ }^{12,13}$ During normal pregnancy, retinal ganglion cells first develop axons that extend toward the optic stalk at 8 weeks gestational age. These axons later form the RNFL. ${ }^{19}$ Provis at $\mathrm{al}^{20}$ investigated the number of axons of the human fetal optic nerve and found that at least $70 \%$ regress during pregnancy. These findings led Mosier et $\mathrm{al}^{12}$ and other investigators ${ }^{14,18}$ to propose the hypothesis that $\mathrm{ONH}$ is the result of excessive RNFL regression. McCulley et $\mathrm{al}^{21}$ suggested that ONH may be the result of retrograde RNFL degeneration from axons not proceeding into the contralateral tract at the chiasm. In this case, one would predict an abnormal asymmetry of the optic nerve rim with greater loss of nasal fibers, because RNFL normally enters into the nasal rim cross-contralaterally at the chiasm. However, we did not observe this pattern.

We found no correlations between optic disc parameters and visual function in patients with ONH. This finding is in agreement with previous clinical observations that there is no relationship between size of the optic nerve and vision in patients with $\mathrm{ONH} .{ }^{13}$ We also found no correlations between visual acuity and RNFL thickness in the temporal area that corresponds to the position of the papillomacular bundle, considered as a possible predictor of visual acuity. ${ }^{22}$ However, most patients with $\mathrm{ONH}$ had visual acuity of $\leq 0.9 \log$ MAR in affected eyes, which limits the range of values with which the relationship can be interpreted.

Currently, it is not clear why some patients with $\mathrm{ONH}$ develop infantile nystagmus forms and others manifest latent nystagmus forms. It would be interesting to investigate a larger cohort of patients with $\mathrm{ONH}$ to see whether a correlation between nystagmus form and clinical/OCT characteristics can be determined.

\section{Foveal Changes}

Optical coherence tomography was able to detect changes in foveal abnormalities in patients with $\mathrm{ONH}$ that were not obvious on fundus examination. Similar observations have been made in patients with foveal hypoplasia, such as albinism and achromatopsia.

This study showed that patients with ONH have significant changes in several retinal layers, including previously described changes in RNFL and GCL, as well as newly observed changes in IPL, OPL, ONL, and IS layers. These changes resemble those observed in foveal hypoplasia, although thinning of RNFL and GCL is not as severe in most types of foveal hypoplasia as that observed in patients with ONH. This is probably due to different mechanisms underlying these changes. Currently, it is difficult to be certain regarding the initial mechanism leading to retinal changes in $\mathrm{ONH}$. Structural macular changes with foveal 


\section{Ophthalmology Volume $\mathbf{-}$, Number $\mathbf{-}$, Month 2015}

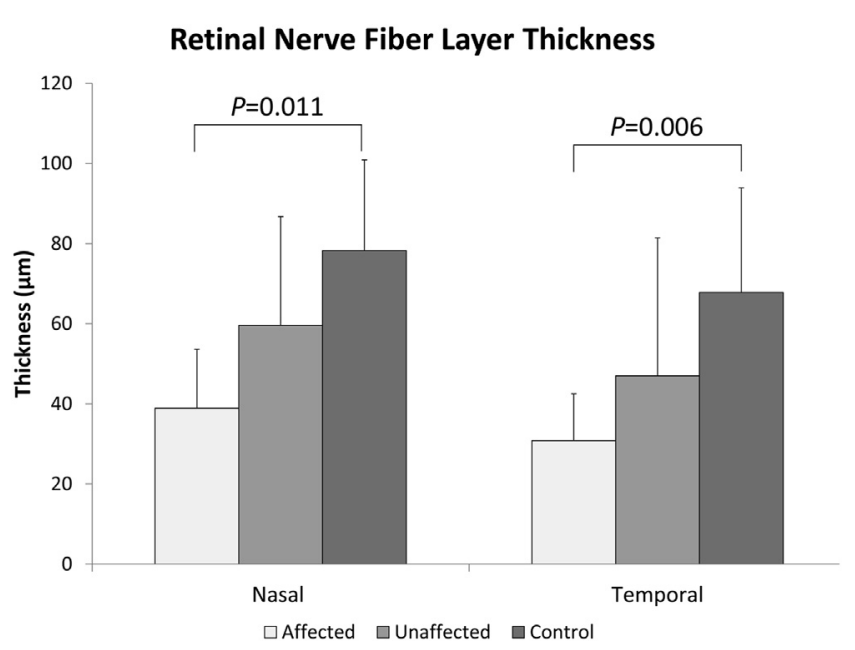

Figure 6. Average thickness of the peripapillary retinal nerve fiber layer (RNFL) in patients with optic nerve hypoplasia (ONH) (affected and unaffected eyes) and healthy controls nasally and temporally from the disc center. Error bars are standard deviations.

involvement could be the result of a more generalized retinal injury or indirectly through transsynaptic degeneration after damage upstream from the retina. Transsynaptic degeneration from the cortex has been suggested as a possible mechanism underlying retinal changes observed in amblyopia. ${ }^{23}$ Alternatively, there could be a simultaneous effect on both the retina and the optic nerve as a result of genetic or environmental perturbation. In agreement with previous histopathologic studies in $\mathrm{ONH}$, we observed thinning of nasal RNFL and temporal and nasal GCL using SD OCT. ${ }^{13,14}$ Retinal nerve fiber layer and GCL loss in the macula have been described in patients with acquired optic nerve atrophy. ${ }^{24-26}$ However, in contrast to histopathologic studies of optic nerve atrophy acquired later in life, we observed that patients with hypoplastic optic nerves also had continuation of the GCL, IPL, and OPL at the fovea and a differently shaped central fovea compared with healthy controls.

Thickening of the central retina was described in several conditions, including amblyopia. ${ }^{23}$ However, simultaneously presented structural changes known as foveal hypoplasia have been described mainly in patients with ocular albinism, PAX6 mutations, and achromatopsia. ${ }^{27}$ During the normal prenatal and early postnatal period (from 25 weeks to $15-45$ months after birth), centrifugal displacement of the inner retinal layers (GCL, IPL, INL) leads to the formation of the foveal pit. At the same time, thickening of the IS and OS layers and widening of the ONL take place. ${ }^{28}$ Therefore, continuation of the IPL, OPL, ONL, and IS in the fovea in ONH suggest arrested foveal development.

Small optic nerve heads have been described in foveal hypoplasia associated with albinism and achromatopsia. Together with our findings in $\mathrm{ONH}$, this indicates that there is an association between foveal maldevelopment and optic nerve development. ${ }^{29,30}$ This is also supported by the significant correlations observed between thickness of retinal layers in the macula and optic nerve parameters.
In $\mathrm{ONH}$, the shape of the fovea is changed compared with controls with more obvious central doming of the ONL (Fig 3). This is different from other forms of foveal hypoplasia in which the shape of the ONL is less pronounced. ${ }^{31}$ When the shape of the ONL is delineated without Henle fibers, by varying pupil entry position of the light with OCT, ${ }^{32}$ a similar peaking shape of the ONL is observed. It is possible that the obvious central doming shape in $\mathrm{ONH}$ is due to reduced Henle fiber layer thickness.

In contrast to optic nerve head parameters, central GCL and OPL in the macula (i.e., indicative of worse foveal maldevelopment) showed a significant positive correlation with SOD. It would be interesting to investigate in future studies, using larger cohorts, whether increased thickness of central GCL and OPL could indicate possible coexisting SOD.

The cause of ONH is not clear. The RNFL and GCL thinning support the theory of ONH occurring because of the retrograde RNFL atrophy during fetal development, as similar changes are described in optic nerve atrophy. The coexistence of SOD and ONH in a subset of patients suggests that SOD may be one possible causal factor leading to ONH. ${ }^{33,34}$

Severe structural changes in the macula and optic nerve of patients with $\mathrm{ONH}$, often associated with midbrain dysgenesis, also may involve the vasculature of the eye. ${ }^{35}$ Therefore, an investigation of the relationship between abnormalities in vascular morphology and structural abnormalities in the retina and optic nerve in ONH would be interesting. All of the optic nerve and macular parameters of unaffected eyes fell midrange between the values for healthy controls and affected eyes. However, there were no significant differences between unaffected eyes and the other 2 groups, possibly because of insufficient numbers of unaffected eyes included. However, there were significant correlations between affected and unaffected eyes for cup depth and retinal thickness of RNFL and IPL. This association suggests a mild degree of $\mathrm{ONH}$ in the unaffected eyes. Small optic nerves were not clinically apparent in the unaffected eyes on fundoscopy, which indicates that OCT is a more sensitive method to detect subtle $\mathrm{ONH}$ than clinical fundus examination.

\section{Study Limitations}

This study represents the largest cohort of patients with $\mathrm{ONH}$ who have been imaged using OCT to date. However, the large variability of the data in the clinically unaffected group may not be adequately represented by the present sample size $(n=10)$. Another limitation of the study was that the analysis was based on single horizontal B-scan images rather than volumetric analysis. This was used because of the presence of nystagmus, often with a vertical component that makes realignment of B-scans unreliable. Consequently, described changes do not represent all the structural abnormalities in patients with $\mathrm{ONH}$ that can be captured by volumetric analysis.

Our study demonstrates significant diagnostic potential of OCT in patients with ONH. Changes in disc and cup 


\section{Pilat et al • Optical Coherence Tomography in $\mathrm{ONH}$}

A
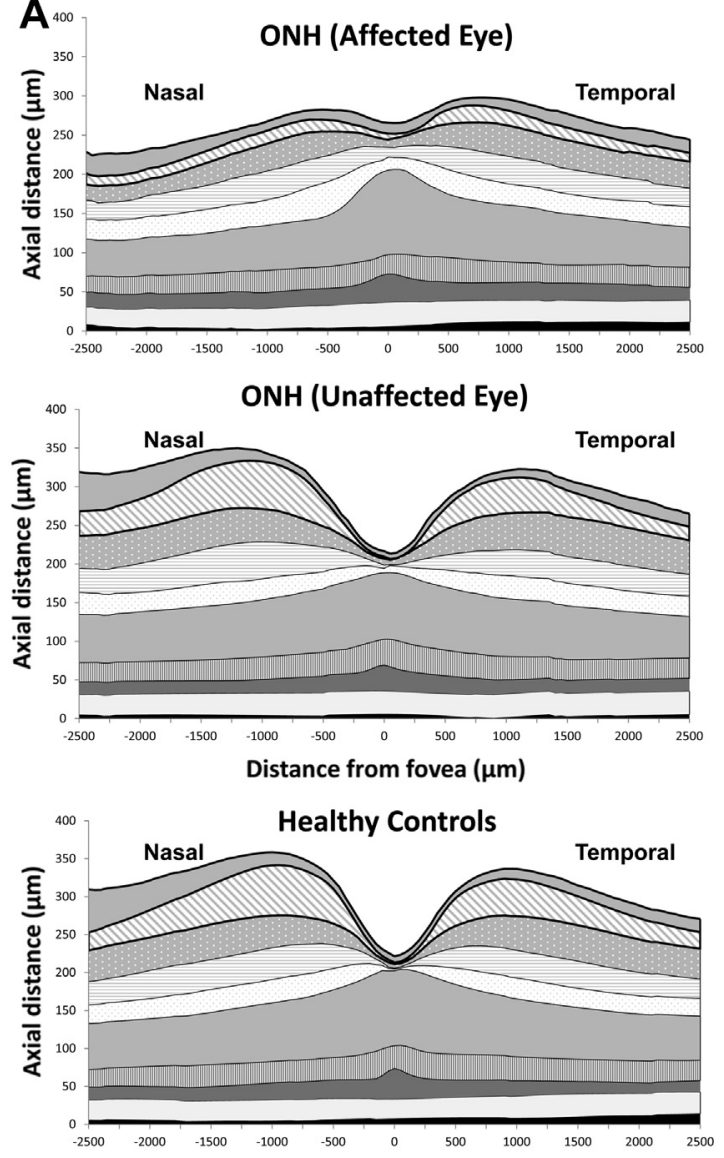

$\square$ RPE $\square$ CC $\square$ OS $\square$ IS $\square$ ONL $\square$ OPL GINL $\square I P L \square G C L \quad \square R N F L$
B
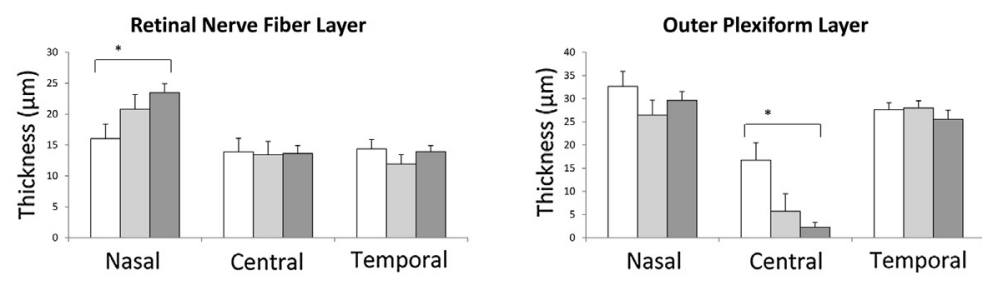
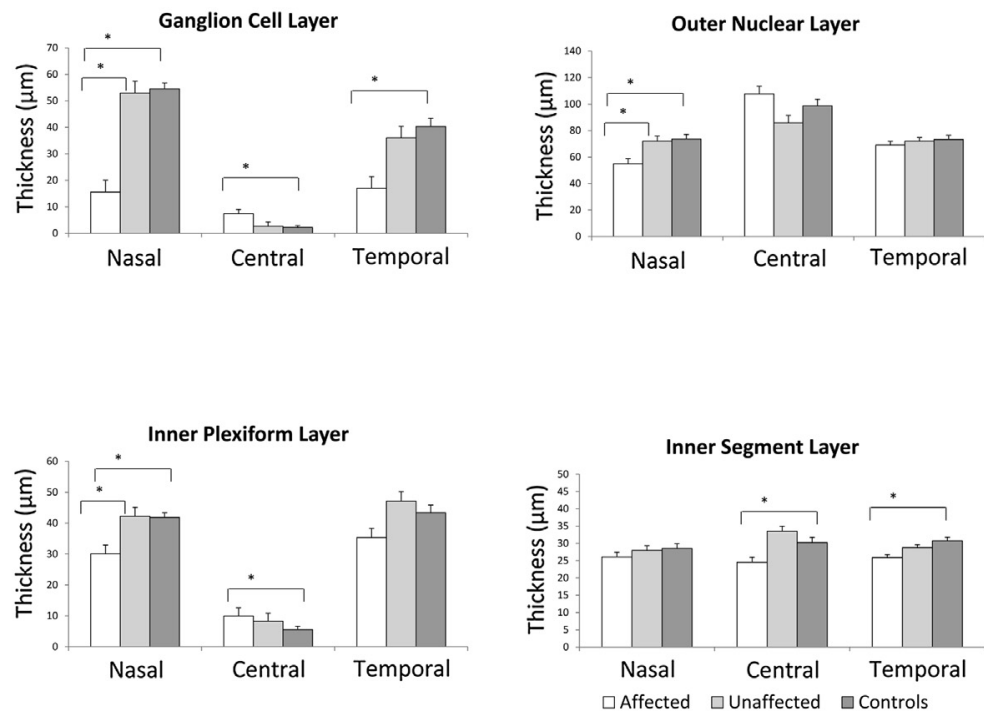

Figure 7. Cross-sectional schematic diagram of individual retinal layers $(\mathrm{A})$ and mean and standard error $(\mu \mathrm{m})$ of the thicknesses of retinal layers when the difference between groups was significant $(\mathrm{B})$ in patients with optic nerve hypoplasia $(\mathrm{ONH})$ (affected and clinically unaffected eyes) and controls. $\mathrm{CC}=$ contact cylinder; $\mathrm{GCL}=$ ganglion cell layer; $\mathrm{INL}=$ inner nuclear layer; $\mathrm{IPL}=$ inner plexiform layer; $\mathrm{IS}=$ inner segment; $\mathrm{ONL}=$ outer nuclear layer; $\mathrm{OPL}=$ outer plexiform layer; $\mathrm{OS}=$ outer segment; RNFL $=$ retinal nerve fiber layer; RPE = retinal pigment epithelium. *Significant difference between groups, $P \leq 0.05$.

diameters and cup depth, as well as thinning of retinal layers and foveal hypoplasia, can help in assessing patients with suspected ONH. High sensitivity and specificity of detecting $\mathrm{ONH}$ on scans indicate that OCT can be useful for the clinical diagnosis. Clinically unaffected eyes show a mild degree of hypoplasia. The macula structure in patients with $\mathrm{ONH}$ is significantly related to visual function and may help to predict visual acuity in infants with $\mathrm{ONH}$.

\section{References}

1. Saadati HG, Hsu HY, Heller KB, Sadun AA. A histopathologic and morphometric differentiation of nerves in optic nerve hypoplasia and Leber hereditary optic neuropathy. Arch Ophthalmol 1998;116:911-6.

2. Patel L, McNally RJ, Harrison E, et al. Geographical distribution of optic nerve hypoplasia and septo-optic dysplasia in Northwest England. J Pediatr 2006;148:85-8.

3. Blohme J, Bengtsson-Stigmar E, Tornqvist K. Visually impaired Swedish children. Longitudinal comparisons 19801999. Acta Ophthalmol Scand 2000;78:416-20.
4. Dattani MT, Robinson IC. HESX1 and septo-optic dysplasia. Rev Endocr Metab Disord 2002;3:289-300.

5. Taranova OV, Magness ST, Fagan BM, et al. SOX2 is a dosedependent regulator of retinal neural progenitor competence. Genes Dev 2006;20:1187-202.

6. Foroozan R. Superior segmental optic nerve hypoplasia and diabetes mellitus. J Diabetes Complications 2005;19:165-7.

7. Stromland K, Pinazo-Duran MD. Ophthalmic involvement in the fetal alcohol syndrome: clinical and animal model studies. Alcohol Alcohol 2002;37:2-8.

8. Borchert M. Reappraisal of the optic nerve hypoplasia syndrome. J Neuroophthalmol 2012;32:58-67.

9. Borchert M, Garcia-Filion P. The syndrome of optic nerve hypoplasia. Curr Neurol Neurosci Rep 2008;8:395-403.

10. Strouthidis NG, Grimm J, Williams GA, et al. A comparison of optic nerve head morphology viewed by spectral domain optical coherence tomography and by serial histology. Invest Ophthalmol Vis Sci 2010;51:1464-74.

11. Jampol LM, Tielsch J. Race, macular degeneration, and the Macular Photocoagulation Study. Arch Ophthalmol 1992;110: 1699-700.

12. Mosier MA, Lieberman MF, Green WR, Knox DL. Hypoplasia of the optic nerve. Arch Ophthalmol 1978;96: $1437-42$. 


\section{Ophthalmology Volume $\mathbf{-}$, Number $\mathbf{n}$, Month 2015}

13. Novakovic P, Taylor DS, Hoyt WF. Localising patterns of optic nerve hypoplasia-retina to occipital lobe. Br J Ophthalmol 1988;72:176-82.

14. Taylor D. Developmental abnormalities of the optic nerve and chiasm. Eye (Lond) 2007;21:1271-84.

15. Epstein AE, Cavuoto KM, Chang TC. Utilizing optical coherence tomography in diagnosing a unique presentation of chiasmal hypoplasia variant of septo-optic dysplasia. J Neuroophthalmol 2014;34:103-4.

16. Moon D, Park TK. Optical coherence tomographic findings in optic nerve hypoplasia. Indian J Ophthalmol 2013;61:596-8.

17. Pilat AV, Proudlock FA, Kumar P, et al. Macular morphology in patients with optic nerve head drusen and optic disc edema. Ophthalmology 2014;121:552-7.

18. Kaur S, Jain S, Sodhi HB, et al. Optic nerve hypoplasia. Oman J Ophthalmol 2013;6:77-82.

19. Barishak YR, Spierer A. Embryology of retina and developmental disorders. In: Hartnett ME, ed. Pediatric Retina. Philadelphia: Lippincott Williams \& Wilkins; 2005:5-9.

20. Provis JM, van Driel D, Billson FA, Russell P. Human fetal optic nerve: overproduction and elimination of retinal axons during development. J Comp Neurol 1985;238: $92-100$.

21. McCulley TJ, Mayer K, Dahr SS, et al. Aniridia and optic nerve hypoplasia. Eye (Lond) 2005;19:762-4.

22. Anderson ML. Monocular nystagmus with sectoral optic nerve hypoplasia in a patient with septo-optic dysplasia. Clin Exp Optom 2009;92:38-41.

23. Bruce A, Pacey IE, Bradbury JA, et al. Bilateral changes in foveal structure in individuals with amblyopia. Ophthalmology 2013; 120:395-403.

24. Gu S, Glaug N, Cnaan A, et al. Ganglion cell layer-inner plexiform layer thickness and vision loss in young children with optic pathway gliomas. Invest Ophthalmol Vis Sci 2014;55:1402-8.

25. Barcella V, Rocca MA, Bianchi-Marzoli S, et al. Evidence for retrochiasmatic tissue loss in Leber's hereditary optic neuropathy. Hum Brain Mapp 2010;31:1900-6.
26. Yamashita T, Miki A, Iguchi Y, et al. Reduced retinal ganglion cell complex thickness in patients with posterior cerebral artery infarction detected using spectral-domain optical coherence tomography. Jpn J Ophthalmol 2012;56:502-10.

27. Thomas MG, Kumar A, Mohammad S, et al. Structural grading of foveal hypoplasia using spectral-domain optical coherence tomography a predictor of visual acuity? Ophthalmology 2011;118:1653-60.

28. Hendrickson A, Possin D, Vajzovic L, Toth CA. Histologic development of the human fovea from midgestation to maturity. Am J Ophthalmol 2012;154:767-778 e2.

29. Thomas MG, Crosier M, Lindsay S, et al. Abnormal retinal development associated with FRMD7 mutations. Hum Mol Genet 2014;23:4086-93.

30. Proudlock F, Mohammad S, Sheth V, et al. Abnormal optic nerve head topography in albinism imaged using high resolution spectral domain-optical coherence tomography. Invest Ophthalmol Vis Sci 2013;54:3232.

31. Mohammad S, Gottlob I, Kumar A, et al. The functional significance of foveal abnormalities in albinism measured using spectral-domain optical coherence tomography. Ophthalmology 2011;118:1645-52.

32. Lujan BJ, Roorda A, Knighton RW, Carroll J. Revealing Henle's fiber layer using spectral domain optical coherence tomography. Invest Ophthalmol Vis Sci 2011;52:1486-92.

33. Brodsky MC, Glasier CM. Optic nerve hypoplasia. Clinical significance of associated central nervous system abnormalities on magnetic resonance imaging. Arch Ophthalmol 1993;111: $66-74$.

34. Ramakrishnaiah RH, Shelton JB, Glasier CM, Phillips PH Reliability of magnetic resonance imaging for the detection of hypopituitarism in children with optic nerve hypoplasia. Ophthalmology 2014;121:387-91.

35. Patton N, Aslam T, Macgillivray T, et al. Retinal vascular image analysis as a potential screening tool for cerebrovascular disease: a rationale based on homology between cerebral and retinal microvasculatures. J Anat 2005;206: 319-48.

\section{Footnotes and Financial Disclosures}

Originally received: October 20, 2014.

Final revision: March 17, 2015.

Accepted: March 17, 2015.

Available online:

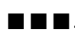

Manuscript no. 2014-1668.

Ophthalmology Group, University of Leicester, Robert Kilpatrick Clinical Sciences Building, Leicester Royal Infirmary, Leicester, United Kingdom.

*Both Anastasia Pilat and Daniel Sibley are first authors.

Financial Disclosure(s):

The author(s) have no proprietary or commercial interest in any materials discussed in this article.

Supported by a Medical Research Council Grant (MR/J004189/1) and the Ulverscroft Foundation.

Author Contributions:

Research design: Pilat, Sibley, McLean, Proudlock, Gottlob

Data acquisition and/or research execution: Pilat, Sibley, McLean
Data analysis and/or interpretation: Pilat, McLean, Gottlob

Obtained funding: Not applicable

Manuscript preparation: Pilat, Sibley, McLean, Proudlock

Abbreviations and Acronyms:

$\mathbf{G C L}=$ ganglion cell layer; $\mathbf{I N L}=$ inner nuclear layer; $\mathbf{I P L}=$ inner plexiform layer; IS = inner segment; $\log$ MAR $=$ logarithm of the minimum angle of resolution; OCT = optical coherence tomography; $\mathbf{O N H}=$ optic nerve hypoplasia; ONHD = optic nerve head drusen; $\mathbf{O P L}=$ outer plexiform layer; $\mathbf{O S}=$ outer segment; $\mathbf{R N F L}=$ retinal nerve fiber layer; RPE = retinal pigment epithelium; $\mathbf{S D}$ OCT $=$ spectral-domain optical coherence tomography; SOD = septo-optic-dysplasia.

Correspondence:

Irene Gottlob, MD, Ophthalmology Group, University of Leicester, Robert Kilpatrick Clinical Sciences Building, Leicester Royal Infirmary, PO Box 65, Leicester, LE2 7LX, UK. E-mail: ig15@le.ac.uk. 